\title{
Mathematical Modeling and Simulation for Optimization of IDEAL-Cell Performance
}

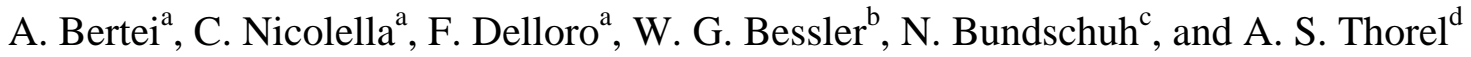 \\ ${ }^{a}$ Dipartimento di Ingegneria Chimica, Università di Pisa, 56126 Pisa, Italy \\ ${ }^{\mathrm{b}}$ German Aerospace Center (DLR), Institute of Technical Thermodynamics \\ 70569 Stuttgart, Germany \\ c VISIMBEL, 70190 Stuttgart, Germany \\ ${ }^{\text {d } C e n t r e ~ d e s ~ M a t e ́ r i a u x, ~ M i n e s-P a r i s T e c h, ~} 91003$ Evry Cedex, France
}

The IDEAL-Cell is an innovative SOFC concept, comprising the anodic part of a proton conducting fuel cell (i.e., anode and protonic electrolyte) and the cathodic part of a solid oxide fuel cell (i.e., cathode and anionic electrolyte), connected through a porous composite central membrane of proton conducting and anion conducting materials where water recombination reaction between protons and oxygen ions occurs. A mathematical model for the description of transport phenomena and reactions in steady-state conditions is presented. The model is based on charge and mass balances in a continuum approach. Simulations are performed considering negligible polarization resistances due to electrochemical activations in order to evaluate the maximum performance of the cell. Simulations show that the IDEAL-Cell performance is comparable to that provided by the current state of the art for proton conducting fuel cells, and it may be further improved by reducing ohmic losses with thinner layers.

\section{Introduction}

The Innovative Dual mEmbrAne fueL-Cell (IDEAL-Cell) is an innovative concept of a solid oxide fuel cell that operates in the range $600-700^{\circ} \mathrm{C}(1)$. It is being developed within an international project funded by the European Commission and the concept was experimentally proven $(2,3)$.

The IDEAL-Cell consists in the junction between the anode and the dense protonic electrolyte of a proton conducting fuel cell (PCFC) with the cathode and the dense anionic electrolyte of a solid oxide fuel cell (SOFC), through a porous composite central membrane (CM) of proton conducting and anion conducting phase. IDEAL-Cell is made of at least five layers of porous (anode, cathode and CM) and dense (electrolytes) materials. Hydrogen is supplied to the anode, wherein it is converted into protons, while air is supplied to the cathode, wherein molecular oxygen is converted into oxygen ions. Protons and oxygen ions migrate through their respective electrolytes towards the CM wherein they react to produce water (Figure 1). 


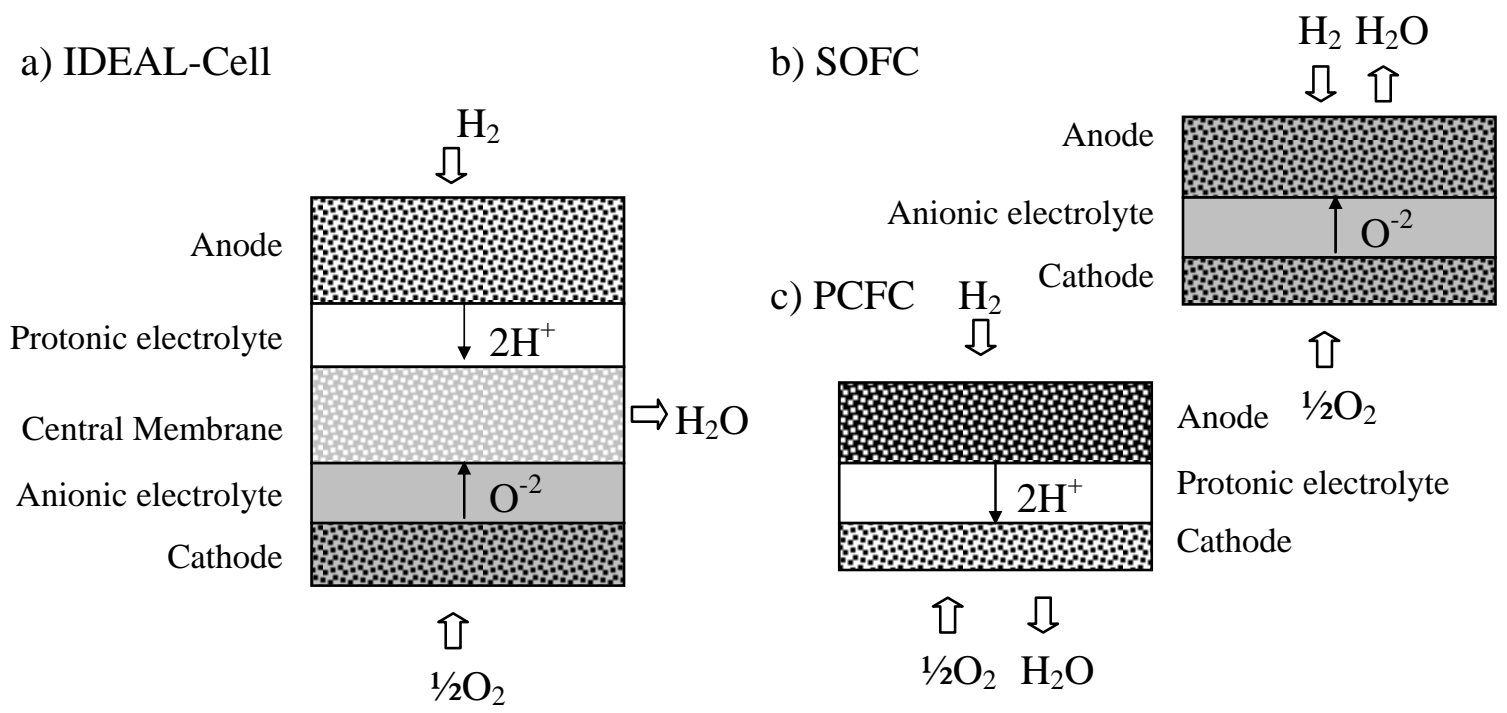

Figure 1. Diagram of the IDEAL-Cell configuration (a) compared with SOFC (b) and PCFC (c) configurations.

Water recombination reaction occurs in the CM, thus avoiding all the severe problems connected to the presence of water at electrodes in both SOFC and PCFC configurations (see Figure 1), in particular the generation of corrosive mixtures with oxygen or hydrogen which may damage interconnect materials (2). Water produced does not dilute the reactants at electrodes, which can therefore be easily recycled; moreover, pure water and heat can be recovered from the CM. Theoretically IDEAL-Cell performance can be improved beyond SOFC and PCFC because anode, cathode and CM are independent. Electrodes and CM can therefore be fully optimized for their basic purposes, that is, the delivery and conversion of reactants into ions for electrodes and production and discharge of water for CM, without any counter-flow of gases. In addition, pressure can be applied at electrodes in order to increase the open circuit voltage (OCV).

In this paper, a mathematical model of charge transfer, mass transport and electrochemical reaction in electrodes and CM is developed. The model is based on charge and mass balances in a continuum approach by using effective transport and kinetic parameters related through percolation theory to morphology and material properties. Despite crude simplifications, this approach has been employed in several modeling studies of composite electrodes yielding valuable results (4-7). The model enables the prediction of steady-state response of the cell under varying operating conditions. In particular, simulations are performed considering high kinetic rates for all electrochemical reactions (i.e., with negligible polarization resistances due to electrochemical activations) in order to evaluate the upper performance limit of the IDEAL-Cell. The main performance indexes investigated are the cell power density, polarization resistances of each layer and maximum pressure inside the CM.

\section{Mathematical Model}

Electrodes and CM are composite cylindrical layers made of different conducting particles randomly distributed and sintered to give enough porosity for gas transport. Each compartment is characterized by its electrochemical reaction: 
$\begin{array}{lrl}\text { Anode } & H_{2(g)} \rightleftharpoons 2 H_{(P C P)}^{+}+2 e_{(e l, a)}^{-} \\ \text {Cathode } & 1 / 2 O_{2(g)}+2 e_{(e l, c)}^{-} \rightleftharpoons O_{(A C P)}^{-2} \\ \text { Central Membrane } & 2 H_{(P C P)}^{+}+O_{(A C P)}^{-2} \rightleftharpoons H_{2} O_{(g)}\end{array}$

Subscripts refer to conducting phases: ACP for anion conducting phase (i.e., the same phase of the anionic electrolyte), el,a for the electronic conductor in the anode while el,c for the cathode and PCP for proton conducting phase (i.e., the same phase of the protonic electrolyte); g represents the gas phase.

Since each reaction involves species in three different phases, reactions must occur in the proximity of the three phase boundary (TPB) among two different conducting particles and gas phase in each composite layer. The reaction can occur at any TPB provided that the gas phase and the two conducting phases are connected either with external sources of reactants or with sinks of products. Thus, only connected TPBs are active for electrochemical reaction. Moreover, transport of molecular and ionic species through their respective connected conducting phases must be considered.

A mechanistic model based on conservation equations for each species involved in each compartment can be derived. The system of balance equations in each layer constitutes the compartment sub-model; the cell model is the union of sub-models. The continuum macroscopic approach is used for electrodes and CM: the porous composite structure is modeled as a continuum phase characterized by uniform effective transport and kinetic parameters, estimated and related to material properties and morphology using percolation theory. This approach enables to use differential conservation equations applied in the whole compartment volume, without distinguishing the three domains, providing a substantial simplification of the modeling and the computing procedures.

\section{Model Assumptions}

The model of the cylindrical cell is based on the following main assumptions:

- steady-state conditions;

- temperature is uniform throughout the cell since heat effects are neglected;

- the morphology of porous composite layers is assimilated to a random packing of monosized spherical particles partly overlapped; effective parameters, estimated by percolation theory, are assumed uniform throughout each layer;

- mixed conduction of charged species is neglected in each conducting phase: ACP conducts only oxygen ions, el,a and el,c only electrons and PCP conducts protons;

- gas transport in each porous layer is described by using the Dusty-Gas Model.

\section{Model Equations}

In each compartment (i.e., electrodes, electrolytes and CM) the sub-model consists in the system of balance equations of all the species involved in the sub-system, expressed for steady-state conditions in a continuum approach. The general conservation equation (i.e., continuity equation) for the generic species $i$ belonging to the compartment $P$ is: 


$$
\nabla \cdot N_{i}^{P}=s_{i}^{P}
$$

where $N_{i}{ }^{P}$ represents the molar flux of $i$ while $s_{i}{ }^{P}$ is the source term (i.e., the rate of generation of $i$ in its respective phase per unit volume of $P$ ). Table I summarizes all model equations for each species in each compartment providing the model of the cell. Model equations for electrolytes are merely represented by:

$$
\begin{array}{lll}
\text { Protonic electrolyte } & \mathrm{H}^{+}{ }_{(\mathrm{PCP})}: & \nabla \cdot N_{H}^{p e}=0 \\
\text { Anionic electrolyte } & \mathrm{O}^{-2}{ }_{(\mathrm{ACP})}: & \nabla \cdot N_{O}^{a e}=0
\end{array}
$$

The model takes into account the presence of inert gases not participating in reactions, indicated as $I$, in each porous layer. Molar source terms are evaluated as the current density exchanged per unit volume divided by the Faraday constant and considering the stoichiometric coefficients of the species as in reactions [1-3]. Current density is represented by its specific term of current per unit of TPB length $i_{P}$ multiplied by the length of connected TPB per unit volume $\lambda_{\text {TPBv }}^{P}$.

TABLE I. Model Equations.

\begin{tabular}{lccccc}
\hline & Anode & CM & Cathode \\
\hline $\mathrm{e}_{(\mathrm{el}, \mathrm{a})}^{-}:$ & $\nabla \cdot N_{e}^{a}=\frac{i_{a} \lambda_{\text {TPBV }}^{a}}{F}$ & $\mathrm{H}_{(\mathrm{PCP})}^{+}:$ & $\nabla \cdot N_{H}^{C M}=-\frac{i_{C M} \lambda_{T P B V}^{C M}}{F}$ & $\mathrm{O}^{-2}(\mathrm{ACP})$ & $\nabla \cdot N_{O}^{c}=\frac{i_{c} \lambda_{\text {TPBV }}^{c}}{2 F}$ \\
$\mathrm{H}_{(\mathrm{PCP})}^{+}:$ & $\nabla \cdot N_{H}^{a}=\frac{i_{a} \lambda_{T P B v}^{a}}{F}$ & $\mathrm{O}^{-2}{ }_{(\mathrm{ACP})}:$ & $\nabla \cdot N_{O}^{C M}=-\frac{i_{C M} \lambda_{T P B V}^{C M}}{2 F}$ & $\mathrm{e}_{(\mathrm{el}, \mathrm{c})}^{-}:$ & $\nabla \cdot N_{e}^{c}=-\frac{i_{c} \lambda_{\text {TPBV }}^{c}}{F}$ \\
$\mathrm{H}_{2(\mathrm{~g})}:$ & $\nabla \cdot N_{h}^{a}=-\frac{i_{a} \lambda_{T P B v}^{a}}{2 F}$ & $\mathrm{H}_{2} \mathrm{O}_{(\mathrm{g})}:$ & $\nabla \cdot N_{w}^{C M}=\frac{i_{C M} \lambda_{T P B v}^{C M}}{2 F}$ & $\mathrm{O}_{2(\mathrm{~g})}:$ & $\nabla \cdot N_{o}^{c}=-\frac{i_{c} \lambda_{\text {TPBV }}^{c}}{4 F}$ \\
$\mathrm{I}_{(\mathrm{g})}:$ & $\nabla \cdot N_{I}^{a}=0$ & $\mathrm{I}_{(\mathrm{g})}:$ & $\nabla \cdot N_{I}^{C M}=0$ & $\mathrm{I}_{(\mathrm{g})}:$ & $\nabla \cdot N_{I}^{c}=0$ \\
\hline
\end{tabular}

Electrochemical Kinetics. Reactions [1-3] are assumed to follow electrochemical kinetics since they involve charged species. Since kinetic studies on the materials used in the IDEAL-Cell have not been developed yet, we assume that the macroscopic kinetics can be represented by the Butler-Volmer expression without mass-transfer effects (8):

$$
i_{P}=i_{0}^{P}\left\{\exp \left[\alpha_{P} \frac{F}{R_{g} T} \eta_{P}\right]-\exp \left[-\left(1-\alpha_{P}\right) \frac{F}{R_{g} T} \eta_{P}\right]\right\}
$$

where $i_{P}$ represents the local current density per unit of TPB length, $i_{0}{ }^{P}$ the exchange current per unit of TPB length, $\alpha_{P}$ the transfer coefficient, $R_{g}$ the gas constant, $T$ the absolute temperature and $\eta_{P}$ the local overpotential. The reaction kinetics is expressed in the direction of water production for positive overpotentials. Table II summarizes all the definitions of local overpotentials and local equilibrium potentials for electrodes and CM. $V_{j}^{P}$ represents the local electric potential of conducting phase $j$ in compartment $P, p_{k}{ }^{P}$ the local partial pressure of molecular component $k$. With the superscript ex we refer to the external gas feeds conditions that surround electrodes or CM. The local equilibrium potential represents the thermodynamic contribution to overpotential yielded by the local partial pressure of gaseous species participating to the compartment reaction. 
TABLE II. Definitions of Local Overpotentials and Local Equilibrium Potentials.

\begin{tabular}{ccc}
\hline Anode & CM & Cathode \\
\hline$\eta_{a}=\Delta V_{a}^{e q}-\left(V_{P C P}^{a}-V_{e l, a}^{a}\right)$ & $\eta_{C M}=\Delta V_{C M}^{e q}-\left(V_{A C P}^{C M}-V_{P C P}^{C M}\right)$ & $\eta_{c}=\Delta V_{c}^{e q}-\left(V_{e l, c}^{c}-V_{A C P}^{c}\right)$ \\
$\Delta V_{a}^{e q}=-\frac{R_{g} T}{2 F} \ln \left(\frac{p_{h}^{a, e x}}{p_{h}^{a}}\right)$ & $\Delta V_{C M}^{e q}=-\frac{R_{g} T}{2 F} \ln \left(\frac{p_{w}^{C M}}{p_{w}^{C M, e x}}\right)$ & $\Delta V_{c}^{e q}=-\frac{R_{g} T}{4 F} \ln \left(\frac{p_{o}^{c, e x}}{p_{o}^{c}}\right)$ \\
\hline
\end{tabular}

Charge Transport. The transport of charged species (i.e., protons, oxygen ions and electrons) in respective conducting phases (i.e., PCP, ACP, el,a and el,c) is assumed to occur by migration following the Ohm law:

$$
N_{j}^{P}=-\frac{\sigma_{C j}^{P}}{Z_{j} F} \nabla V_{C j}^{P}
$$

where $N_{j}^{P}$ is the molar flux of the charged species $j$ in the compartment $P, z_{j}$ its charge, $\sigma_{C j}^{P}$ the conductivity of the conducting phase $C j$ that transports $j$ and $V^{P}{ }_{C j}$ the electric potential of the conducting phase $C j$. For electrolytes the conductivity $\sigma_{C j}^{P}$ is the conductivity of dense material; for electrodes and CM effective conductivity (marked with eff) of each conducting phase must be considered.

Mass Transport. In porous layers, that is, electrodes and CM, molecular species are transported in the pores of gas phase. Mass transport is described according to the DustyGas Model for transition region (9), considering convection and diffusion (both ordinary and Knudsen diffusion) of reacting molecular species and inert gas. The net flux $N_{i}^{P}$ of molecular species $i$ in the porous compartment $P$ is described by the relationship:

$$
\frac{N_{i}^{P}}{D_{i}^{k-e f f, P}}+\sum_{\substack{j=1 \\ j \neq i}}^{n} \frac{x_{j}^{P} N_{i}^{P}-x_{i}^{P} N_{j}^{P}}{D_{i j}^{e f f, P}}=-\frac{P^{P}}{R_{g} T} \nabla x_{i}^{P}-\frac{x_{i}^{P}}{R_{g} T}\left(1+\frac{B^{P}}{D_{i}^{k-e f f, P}}\right) \nabla P^{P}
$$

where $P^{P}$ is the local total pressure, $x_{i}$ the molar fraction of $i, n$ the number of components in gas phase. $D_{i}^{k \text {-eff,P }}$ and $D_{i j}{ }^{\text {eff,P }}$ represent, respectively, the effective Knudsen diffusivity of $i$ and the effective ordinary bulk diffusivity for the pair $i-j$, while $B^{P}$ is the effective permeability coefficient. Such parameters depend on both physical properties of gas mixtures and morphological properties of the porous layers, in particular on the mean pore diameter $D^{P}$ and the tortuosity of gas phase $\tau^{P}$. For binary mixtures, as it happens in our case with a single reacting molecular species and a single inert gas in each compartment, fluxes expressions can be derived explicitly from Eq. [9] as a function of total pressure and molar fraction of one of the two species.

Effective Morphological Parameters. Electrodes and CM are assimilated to a random binary packing of monosized spherical particles, namely $k$ and $l$, i.e., the two conducting phases inside the compartment $P$. Assuming that gas phase is entirely connected (10), the length of connected TPB per unit volume $\lambda^{P}{ }_{T P B v}$ between conducting phases (i.e., $k$ and $l$ ), the length of connected TPB per unit of electrolyte surface area $\lambda^{P}{ }_{\text {TPBs-k }}$ (i.e., the TPB created by percolating $k$ particles that reach the electrolyte, constituted by $l$ phase) and the mean pore diameter $D^{P}$ are estimated using percolation theory according to (11-13). 
Effective conductivities are estimated by computer simulations of random packings of overlapping spheres (13). The microstructure is generated by assigning a random position to $k$ and $l$ particles in a body centered cubic structure according to their relative volume fraction, effective conductivities of phases are evaluated by using the finite element method to solve the Ohm law within the conducting phases. Simulated effective conductivities obtained are in good agreement with similar studies (14).

\section{$\underline{\text { Boundary Conditions }}$}

Table III resumes all boundary conditions at each compartment interface along the axial direction for each sub-model; in this table $\hat{n}$ represents the normal unitary vector exiting from the surface. Reactions at electrolytes interfaces are considered and in each sub-model a compartment overpotential $\eta^{P}$ is imposed. The overall cell model considers the continuity of the cell current density.

TABLE III. Boundary Conditions for Sub-models at Compartments Interfaces (Axial Direction).

\begin{tabular}{|c|c|c|c|c|}
\hline Compartment & Interface & Boundary Conditions & Interface & Boundary Conditions \\
\hline \multirow{4}{*}{ Anode } & \multirow{4}{*}{$\begin{array}{c}\text { Anode } \\
\text { External } \\
\text { Atmosphere }\end{array}$} & $V_{e l, a}^{a}=\eta^{a}$ & \multirow{4}{*}{$\begin{array}{l}\text { Protonic } \\
\text { Electrolyte } \\
\text { Interface }\end{array}$} & $\hat{n} \cdot N_{e}^{a}=-\frac{\lambda_{T P B S-e l, a}^{a} i_{a}}{F}$ \\
\hline & & $\hat{n} \cdot N_{H}^{a}=-\frac{\lambda_{T P B s-P C P}^{a} i_{a}}{F}$ & & $V_{P C P}^{a}=0$ (reference) \\
\hline & & $P^{a}=P^{a, e x}$ & & $\hat{n} \cdot N_{h}^{a}=\frac{\lambda_{\text {TPBs }-e l, a}^{a} i_{a}}{\rho \cdot}$ \\
\hline & & $x_{h}^{a}=x_{h}^{a, e x}$ & & $\hat{n} \cdot N_{I}^{a}=0$ \\
\hline \multirow{5}{*}{$\mathbf{C M}$} & & $V_{P C P}^{C M}=\eta^{C M}$ & \multirow{5}{*}{$\begin{array}{l}\text { Anionic } \\
\text { Electrolyte } \\
\text { Interface }\end{array}$} & $\hat{n} \cdot N_{H}^{C M}=\frac{\lambda_{T P B s-P C P}^{C M} i_{C M}}{F}$ \\
\hline & Protonic & $\hat{n} \cdot N_{O}^{C M}=\frac{\lambda_{T P B S-A C P}^{C M} i_{C M}}{2 F}$ & & $V_{A C P}^{C M}=0$ (reference) \\
\hline & $\begin{array}{l}\text { Electrolyte } \\
\text { Interface }\end{array}$ & $\hat{n} \cdot N^{C M}=-\frac{\lambda_{T P B S-A C P}^{C M} i_{C M}}{2 F}$ & & $\hat{n} \cdot N^{C M}=-\frac{\lambda_{T P B s-P C P}^{C M} i_{C M}}{.0}$ \\
\hline & & $n \cdot N_{w}=-\frac{2 F}{2 F}$ & & $n \cdot N_{w}=-\frac{2 F}{2 F}$ \\
\hline & & $\hat{n} \cdot N_{I}^{C M}=0$ & & $\hat{n} \cdot N_{I}^{C M}=0$ \\
\hline \multirow{4}{*}{ Cathode } & & $V_{A C P}^{c}=\eta^{c}$ & \multirow{4}{*}{$\begin{array}{l}\text { Cathode } \\
\text { External } \\
\text { Atmosphere }\end{array}$} & $\hat{n} \cdot N_{O}^{c}=-\frac{\lambda_{T P B S-A C P}^{c} i_{c}}{2 F}$ \\
\hline & Anionic & $\hat{n} \cdot N_{e}^{c}=\frac{\lambda_{\text {TPBs-el,c }}^{c} i_{c}}{F}$ & & $V_{e l, c}^{c}=0$ (reference) \\
\hline & $\begin{array}{l}\text { Electrolyte } \\
\text { Interface }\end{array}$ & $\hat{n} \cdot N_{o}^{c}=\frac{\lambda_{T P B s-e l, c_{c}^{c}}^{c} i_{c}}{4 F}$ & & $P^{c}=P^{c, e x}$ \\
\hline & & $\hat{n} \cdot N_{I}^{c}=0$ & & $x_{o}^{c}=x_{o}^{c, e x}$ \\
\hline
\end{tabular}

Radial boundary conditions for electrodes and electrolytes are trivial: no-flux conditions are assumed for all species. For the CM, since water outflux follows the radial direction (Figure 1a), at the interface between CM and outer atmosphere, no-flux conditions are assumed for charged species while external conditions for pressure and molar fraction of water are imposed as, respectively, $P^{C M}=P^{C M, e x}$ and $x_{w}{ }^{C M}=x_{w}{ }^{C M, e x}$. 


\section{Simplified Approaches}

Several simplifications can be adopted when particular requirements are satisfied.

For porous layers, if mass transport in gas phase is fast enough it can be neglected, that is, within the compartment uniform conditions for pressure and gas molar fractions, equal to external conditions, can be assumed. This situation happens for electrodes because mass transport occurs along the axial coordinate (see Figure 1) where the thickness is small. Then, in the limit of high kinetics rates, polarization resistance assumes a simple form as:

$$
R_{p}^{a}=\frac{t_{a}}{\sigma_{e l, a}^{e f f, a}+\sigma_{P C P}^{e f f, a}} \text { and } R_{p}^{c}=\frac{t_{c}}{\sigma_{A C P}^{e f f, c}+\sigma_{e l, c}^{e f f, c}}
$$

where $t_{a}$ and $t_{c}$ are respectively anode and cathode thicknesses. Thus, anode and cathode polarization resistances are merely function of material properties and morphology, i.e., independent on the current density and external conditions. A similar situation happens for electrolytes where the model predicts a constant resistance equal to $t_{p e} / \sigma_{P C P}$ or $t_{a e} / \sigma_{A C P}$.

Since water outflux from the CM occurs along the radial direction, simulations show that pressure effects are expected to be significant within the CM. In order to reduce the pressure increase, the $\mathrm{CM}$ is designed with a structure with sub-cells and channels to promote water outflux (Figure 2). It is assumed that gas transport within channels is fast enough to set outer atmosphere conditions at each sub-cell external surface area. In this way, we do not solve the problem of transport of gases in channels and the sub-model of the CM, as reported in Table I, is applied only to a single sub-cell.

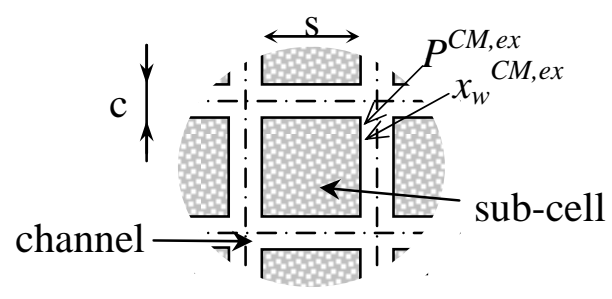

Figure 2. Central membrane structure with sub-cells and channels: external conditions are considered in each channel.

\section{Results and Discussion}

Simulations are performed considering high kinetic rates in order to evaluate the upper performance limit of the cell. Moreover, morphological properties are chosen in order to increase effective conductivities: porosity of each porous layer is set to $35 \%$ and contact angles $\theta^{P}$ to $30^{\circ}$ despite the actual state of the art does not reach these conditions yet (3). Due to the simplified approaches mentioned previously, only the CM sub-model needs a numerical solution. The CM sub-model is solved by using Comsol Multiphysics 3.5 with a finite element method. Model parameters are listed in Table IV, parameters for mass transport are taken from (17). Thicknesses reported in Table IV correspond to the best available shaping technologies for materials used in IDEAL-Cell. Transfer coefficients $\alpha_{P}$ are set to 0.5 , tortuosities $\tau^{p}$ are assumed equal to 2.083 (13). 
TABLE IV. Model Parameters.

\begin{tabular}{lc}
\hline & Value \\
\hline Operating Conditions & $600{ }^{\circ} \mathrm{C}$ \\
$\quad$ Temperature, $T$ & $1-1-1 \mathrm{~atm}$ \\
$\quad$ Outside Pressures, $P^{a, e x}-P^{C M, e x}-P^{c, e x}$ & $0.97-0.05-0.21$ \\
$\quad$ Outside Molar Fractions, $x_{h}^{a, e x}-x_{w}{ }^{C M, e x}-x_{o}^{c, e x}$ & $\mathrm{H}_{2} \mathrm{O}-\mathrm{N}_{2}-\mathrm{N}_{2}$ \\
$\quad$ Inert gases, anode $-C M-$ cathode & \\
Cell Design Parameters & $500-500-50-30-30 \mu \mathrm{m}$ \\
$\quad$ Thicknesses, $t_{a}-t_{p e}-t_{C M}-t_{a e}-t_{c}$ & $0.2 / 0.2-0.2 / 0.2-0.2 / 0.2 \mu \mathrm{m}$ \\
$\quad$ Particles Size, $d_{e l, a}^{a} / d_{P C P}^{a}-d_{P C P}^{C M} / d_{A C P}^{C M}-d_{A C P}^{c} / d_{e l, c}^{c}$ & $50 / 50-50 / 50-50 / 50 \% \mathrm{vol}$. \\
$\quad$ Composition, $\psi_{e l, a}^{a} / \psi_{P C P}^{a}-\psi_{P C P}^{C M} / \psi_{A C P}^{C M}-\psi_{A C P}^{c} / \psi_{e l, c}^{c}$ & $35-35-35 \%$ \\
$\quad$ Porosity, $\phi \phi^{a}-\phi^{C M}-\phi^{f}$ & \\
Material properties & $\mathrm{Ni}-\mathrm{BCY} 15-\mathrm{YDC} 15-\mathrm{LSCF} 48$ \\
$\quad$ Materials, $e l, a-P C P-A C P-e l, c$ & $2.34 \cdot 10^{6}(15)-6.6-1.1-4.57(16) \mathrm{S} / \mathrm{m}$ \\
$\quad$ Conductivities, $\sigma_{e l, a}-\sigma_{P C P}-\sigma_{A C P}-\sigma_{e l, c}$ &
\end{tabular}

Figure 3a shows the simulated polarization curve for a sub-cell dimension $s=2 \mathrm{~mm}$ (see Figure 2). The linear dependence of the voltage at terminals $V$ as a function of the cell current density $i_{\text {tot }}$ is expected since activation resistances are negligible because high exchange currents are used. The maximum cell power density Pow (expressed per unit of sub-cell cross section area in this and in following figures) is equal to $113.6 \mathrm{~mW} / \mathrm{cm}^{2}$ at $i_{\text {tot }}=2000 \mathrm{~A} / \mathrm{m}^{2}$. Figure 3b shows the contributions of each layer to the cell polarization resistance: the main losses are due to the CM and the protonic electrolyte, i.e., the thickest and least conductive layers.

a)

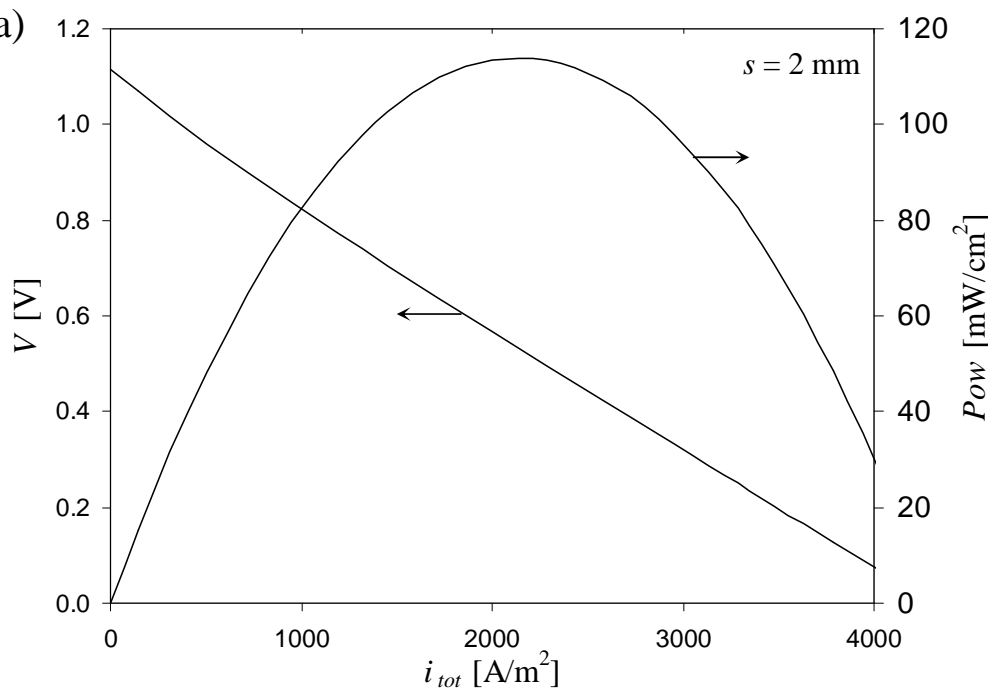

b) Anode
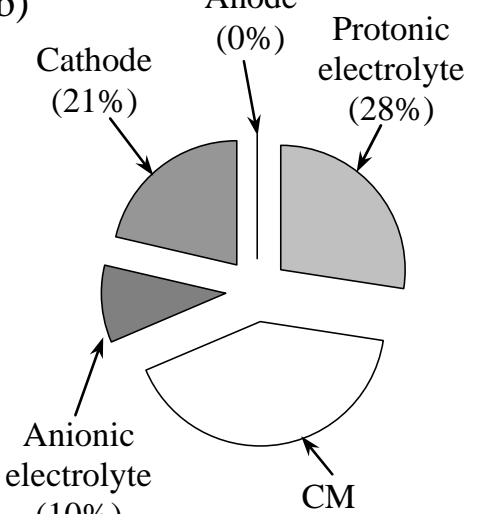

$(10 \%)$

$$
R_{p}^{\text {cell }}=2.74 \cdot 10^{-4} \Omega m^{2}
$$

Figure 3. a) Simulated polarization curve; b) contributions to cell polarization resistance at $i_{\text {tot }}=2000 \mathrm{~A} / \mathrm{m}^{2}$.

The variation of CM polarization resistance as a function of sub-cell dimension $s$ is plotted in Figure 4 for $i_{\text {tot }}=2000 \mathrm{~A} / \mathrm{m}^{2}$. CM polarization resistance decreases as $s$ decreases reaching an asymptotic limit value of $7.3 \cdot 10^{-5} \Omega \mathrm{m}^{2}$. As a consequence, cell power density increases up to the asymptotic limit of $129.4 \mathrm{~mW} / \mathrm{cm}^{2}$. At the same time, the decrease of sub-cell dimension leads to a decrease of CM maximum pressure (Figure 5) since water produced, driven outside by both concentration and pressure gradients, has to cover a smaller length in the radial direction. In the same figure, the ratio between the 
absolute value of the maximum $\mathrm{CM}$ local equilibrium potential and the overpotential applied is reported as a function of $s$. According to Table II, the increase of $\left|\Delta V^{e q}{ }_{C M, \max }\right|$ with $s$ is obvious since it depends on local CM partial pressure of water that increases as $s$ increases.

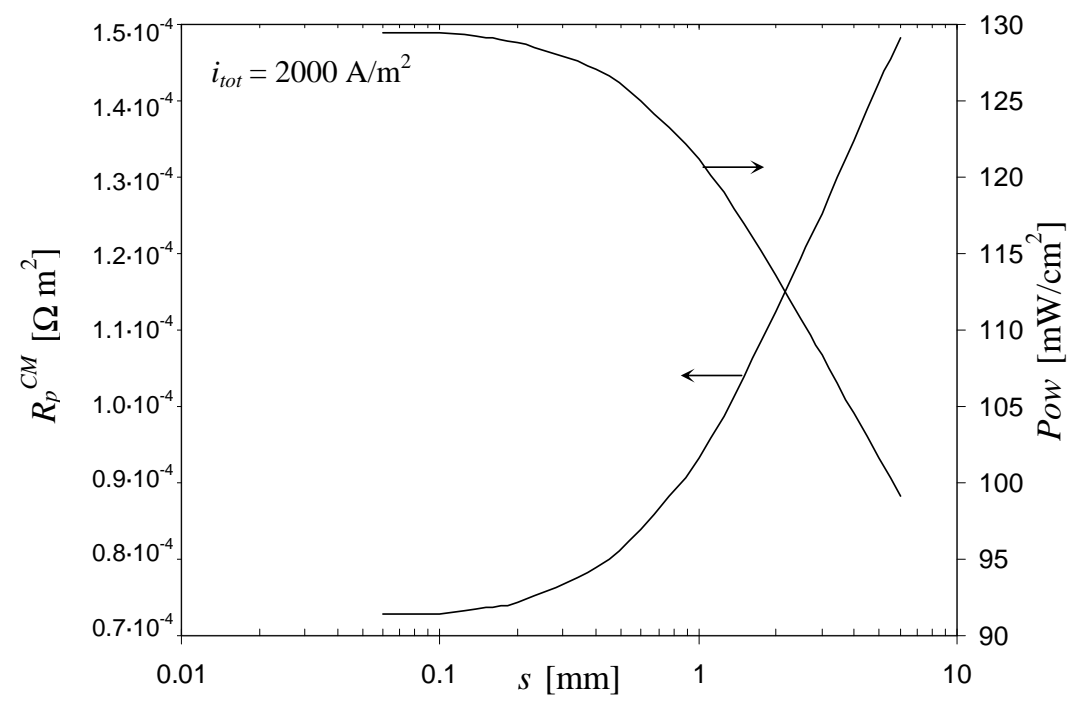

Figure 4. CM polarization resistance and cell power density as a function of sub-cell dimension at $i_{\mathrm{tot}}=2000 \mathrm{~A} / \mathrm{m}^{2}$.

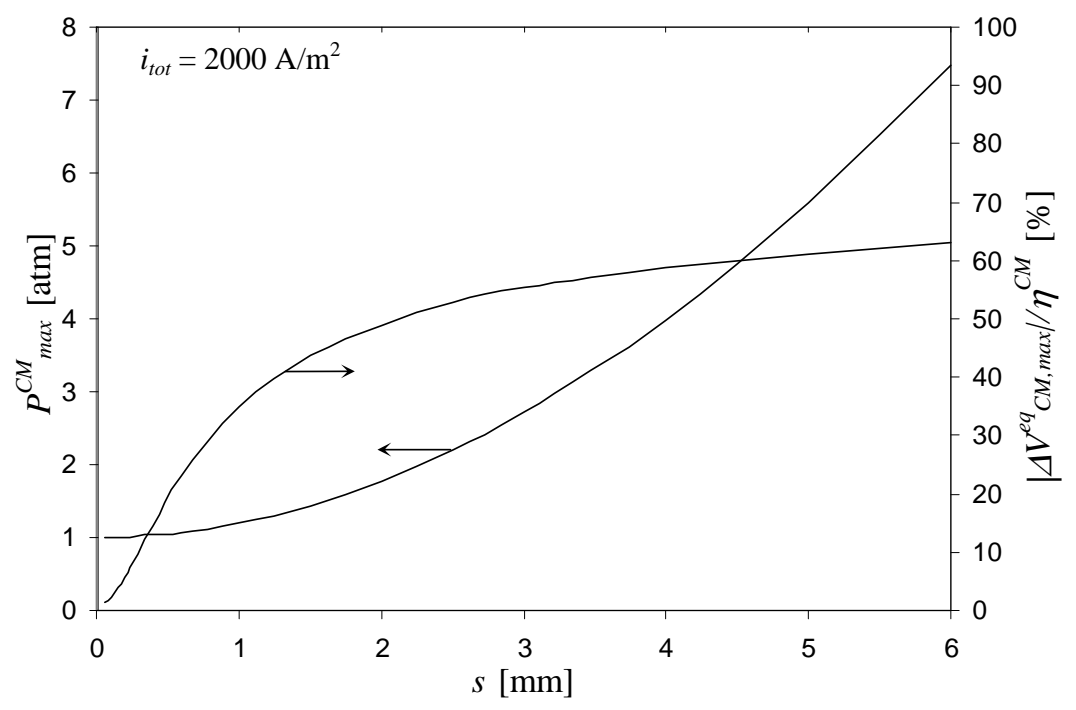

Figure 5. CM maximum pressure and ratio between the absolute value of the maximum CM equilibrium potential and the CM overpotential as a function of sub-cell dimension.

This last behavior is useful to explain why CM polarization resistance decreases as sub-cell dimension decreases. It should be demonstrated that when activation resistances are negligible, water formation occurs merely at CM-electrolytes interfaces. Thus, CM polarization resistance is yielded by the transport of part of oxygen ions (i.e., the part that has not reacted at CM-anionic electrolyte interface) from anionic electrolyte towards CM-protonic electrolyte interface through CM thickness and the transport of part of protons from protonic electrolyte to $\mathrm{CM}$-anionic electrolyte interface through $\mathrm{CM}$ thickness. The transport of the two charged species is not uniform within the sub-cell thickness, but it is localized mainly on the external region close to outer atmosphere 
because here its driving forces, i.e., the gradients of ACP or PCP potential along the axial direction, are bigger than in the middle of the sub-cell as represented in Figure 6.

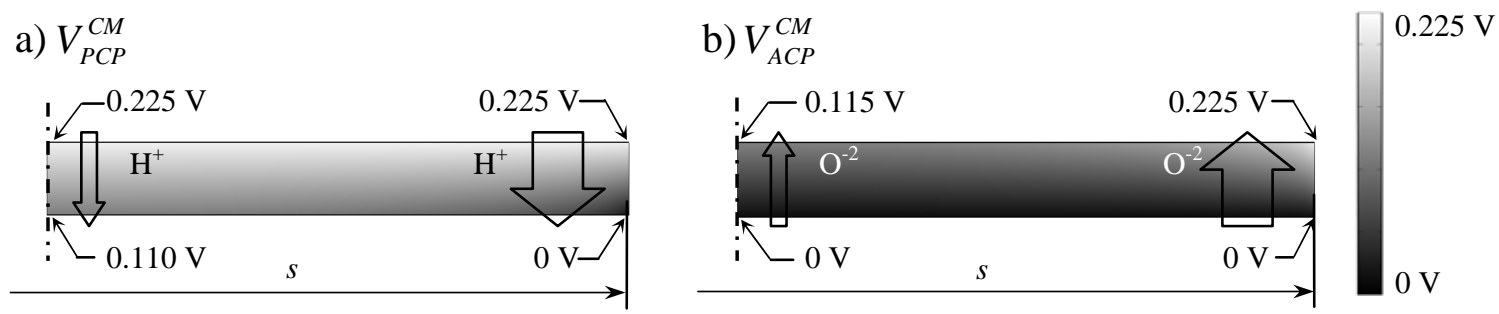

Figure 6. a) PCP potential and b) ACP potential profiles in a sub-cell slice for $s=2 \mathrm{~mm}$ and $i_{\text {tot }}=2000 \mathrm{~A} / \mathrm{m}^{2}$ (corresponding to $\eta^{C M}=0.225 \mathrm{~V}$ ).

It is the local equilibrium potential that determines PCP and ACP potential profiles, thus, since $\left|\Delta V^{e q}{ }_{C M}\right|$ increases with $s, C M$ polarization resistance increases due to the nonuniform passage of current. The increase of the local absolute equilibrium potential forces the current to pass close to the external perimeter of the sub-cell providing an increase of CM polarization resistance relative to the sub-cell cross section area.

Several design considerations are obtained as a result from the present simulations:

- the main losses are due to thickest and least conductive layers, that is, protonic electrolyte and CM (Figure 3). Cell performance could reasonably be improved by reducing thicknesses of layers and increasing effective conductivities;

- simulations show that maximum pressure and polarization resistance decrease as sub-cell dimensions decrease (Figures 4-5). Mechanical constraints and cell performance are both satisfied by decreasing sub-cell dimension;

- CM polarization resistance and cell power density have been calculated per unit of sub-cell cross section area. If referred to the effective area $(s+c)^{2}$ instead of $s^{2}$, i.e., considering also channel diameter (see Figure 2), cell power density is reduced by the ratio $s^{2} /(s+c)^{2}$, especially for small values of sub-cell dimension.

Finally, we may conclude that an optimal choice of the dimensions of channel diameter $c$ (that will assure an efficient water transport within channels) and of sub-cell dimension $s$ (that will affect CM polarization resistance and water pressure within subcell) must be achieved as a compromise between performance and mechanical stress due to pressure build-up.

\section{Conclusions}

A mathematical model for the description of transport phenomena and reactions in the IDEAL-Cell under steady-state conditions, based on charge and mass balances in a continuum approach, has been presented. The model is used to estimate the upper performance limit of the cell assuming negligible activation overpotentials and considering the sub-cell dimension as a design variable.

Simulations show that maximum pressure and CM polarization resistance decrease as sub-cell dimension decreases. Nevertheless, the optimal sub-cell dimension should be chosen as a compromise of mechanical constraints and performance considering also the 
channel diameter, that is, considering the ratio of CM active over CM channel area. The estimated IDEAL-Cell performance is comparable with the current state of the art for PCFC and it may be further improved by reducing ohmic losses with thinner layers.

Additional design parameters such as channel diameter, porosities, thicknesses of layers, particles diameter and compositions further affect IDEAL-Cell performance. The assessment and optimization of these parameters will be subject of future investigations.

\section{Acknowledgments}

This research has received funding from the European Community's FP7 Programme under grant agreement No 213389.

\section{References}

1. See www.ideal-cell.eu

2. A. S. Thorel, A. Chesnaud, M. Viviani, A. Barbucci, S. Presto, P. Piccardo, Z. Ilhan, D. E. Vladikova and Z. Stoynov, in Solid Oxide Fuel Cells 11/2009, S. C. Singhal and H. Yokokawa Editors, p. 753, The Electrochemical Society Proceedings Series, Pennington, NJ (2009).

3. S. Presto, A. Barbucci, M. Viviani, Z. Ilhan, S. A. Ansar, D. Soysal, A. S. Thorel, J. Abreu, A. Chesnaud, T. Politova, K. Przybylski, J. Prazuch, Z. Zhao, D. Vladikova and Z. Stoynov, in Solid Oxide Fuel Cells 11/2009, S. C. Singhal and H. Yokokawa Editors, p. 773, The Electrochemical Society Proceedings Series, Pennington, NJ (2009).

4. P. Costamagna, P. Costa and V. Antonucci, Electrochim. Acta, 43, 375 (1998).

5. H. Zhu and R. J. Kee, J. Electroch. Soc., 155, B715 (2008).

6. B. Kenney and K. Karan, Solid State Ionics, 178, 297 (2007).

7. C. Nicolella, A. Bertei, M. Viviani and A. Barbucci, J. Appl. Electrochem., 39, 503 (2009).

8. A. J. Bard and L. R. Faulkner, Electrochemical Methods: Fundamentals and Applications $2^{\text {nd }}$ ed., p. 100, John Wiley \& Sons, New York (2001).

9. D. Arnošt and P. Schneider, Chem. Eng. J., 57, 91 (1995).

10. B. Kenney, M. Valdmanis, C. Baker, J. G. Pharoah and K. Karan, J. Power Sources, 189, 1051 (2009).

11. A. Bertei and C. Nicolella, Powder Tech., submitted (2010).

12. A. Bertei and C. Nicolella, J. Power Sources, submitted (2010).

13. A. Bertei, Mathematical Modelling of an Innovative Solid Oxide Fuel Cell, pp. 82, 66, 104, Master Degree Thesis, University of Pisa (2010).

14. H. W. Choi, A. Berson, B. Kenney, J. G. Pharoah, S. Beale and K. Karan, in Solid Oxide Fuel Cells 11/2009, S. C. Singhal and H. Yokokawa Editors, p. 1341, The Electrochemical Society Proceedings Series, Pennington, NJ (2009).

15. J. H. Nam and D. H. Jeon, Electrochimica Acta, 51, 3446 (2006).

16. G. C. Kostogloudis and C. Ftikos, Solid State Ionics, 126, 143 (1999).

17. B. Todd and J. B. Young, J. Power Sources, 110, 186 (2002). 